\title{
Optical spin polarization and exchange interaction in doubly charged InAs self-assembled quantum dots
}

\author{
V. K. Kalevich,,${ }^{1,2, *}$ I. A. Merkulov, ${ }^{1}$ A. Yu. Shiryaev, ${ }^{1}$ K. V. Kavokin,,${ }^{1}$ M. Ikezawa, ${ }^{2}$ T. Okuno,${ }^{2}$ P. N. Brunkov, ${ }^{1}$ \\ A. E. Zhukov, ${ }^{1}$ V. M. Ustinov, ${ }^{1}$ and Y. Masumoto ${ }^{2}$ \\ ${ }^{1}$ Ioffe Physico-Technical Institute, St. Petersburg 194021, Russia \\ ${ }^{2}$ Institute of Physics, University of Tsukuba, Tsukuba 305-8571, Japan
}

(Received 19 November 2004; revised manuscript received 29 April 2005; published 14 July 2005)

\begin{abstract}
The work is an experimental study of optical spin polarization in InAs/GaAs quantum dots (QDs) with two resident electrons or holes. A capture of a photogenerated electron-hole pair into such a QD creates a negative or positive tetron (doubly charged exciton). Spin polarization was registered by the circular polarization of the QD photoluminescence (PL). The spin state was found to be radically different in the dots with the opposite sign of the charge. Particularly, under excitation in a GaAs barrier, the polarization of the ground-state PL is negative (relative to the polarization of exciting light) in the negatively charged QDs and positive in the positively charged QDs. With increasing excitation intensity, the negative polarization rises from zero up to a saturation level, while the positive polarization decreases. The negative polarization increases in weak magnetic fields applied in Faraday geometry; however, it is suppressed in strong fields. The positive polarization always increases as a function of magnetic field. We propose a theoretical model that qualitatively explains the experimental results.
\end{abstract}

DOI: 10.1103/PhysRevB.72.045325

PACS number(s): 72.25.Fe, 72.25.Rb, 73.21.La, 78.55.Cr

\section{INTRODUCTION}

When one passes from three-dimensional to zerodimensional objects, the role of different mechanisms of spin relaxation changes significantly. When analyzing different mechanisms of spin relaxation for the carriers localized in semiconductor quantum dots (QDs), it is convenient to use the analogy with the spin relaxation of deep paramagnetic centers. ${ }^{1}$ The relaxation due to spin-orbital interaction gets suppressed, ${ }^{2}$ while the exchange interaction intensifies and becomes anisotropic, ${ }^{3,4}$ and the relaxation by nuclei also gets stronger. ${ }^{5}$ An anisotropic exchange interaction of electrons and holes induced by the dot asymmetry can split the exciton radiative doublet and destroy spin polarization of QD excitons., ${ }^{3,4}$ The anisotropic exchange can be strongly suppressed in charged QDs containing an odd number of carriers (including photocreated electron-hole pairs), since such QDs have half-integer spin and the anisotropic exchange does not split spin states due to the Kramers theorem. ${ }^{6}$ Indeed, a longliving spin orientation has been observed in negatively charged InAs, ${ }^{7}$ InP, ${ }^{8}$ and $\mathrm{ZnSe}$ (see Ref. 9) QDs containing one or three resident electrons. As the Kramers theorem is not applicable to the complexes comprising an even number of particles, the question of the spin conservation in QDs with an even number of resident carriers remained, so far, open.

Generally, an analysis of the exchange interaction is significantly complicated by increasing the number of resident carriers in a QD. However, the situation is radically simplified in a QD with two resident carriers. The matter is that in such a QD the ground state of majority carriers is a singlet with a zero spin. Therefore, the exchange interaction of the four-particle complex (tetron) containing two resident carriers and a photoexcited electron-hole pair reduces to the exchange interaction of the photoexcited pair. The latter is the analog of an ordinary exciton. But in contrast with an ordinary exciton, in the tetron one of the particles coupled by the exchange interaction (hole or electron) is in the ground state, while the other one (electron or hole) is in the first excited state. This provides a possibility to study an exchange interaction of particles located at different orbital states. In this work, we study optical spin polarization in QDs with two resident electrons or holes.

\section{SAMPLES AND EXPERIMENTAL RESULTS}

We studied the molecular-beam expitaxy (MBE) grown structures containing ten layers of self-assembled InAs/GaAs QDs separated by 30-nm-thick GaAs barriers. The dot density in a layer of about $0.5 \times 10^{11} \mathrm{~cm}^{-2}$, base diameter of $\approx 15 \mathrm{~nm}$, and height of $\approx 5 \mathrm{~nm}$ was found from the TEM studies (for review see, e.g., Refs. 10 and 11). The dots are positively or negatively charged due to delta doping by $\mathrm{Si}$ or Be, respectively. The delta-doping layers are located $15 \mathrm{~nm}$ below each QD layer. The dopant density was equal to the double QD density producing nominally two resident electrons or holes per dot on average. The MBE grown structure with a single layer of InAs QDs embedded into the middle of the $i$ layer of the $n-i-n$ GaAs matrix was also studied. GaAs barriers, cladding the $i$ layer, are doped by $\mathrm{Si}$ with concentration of $2 \times 10^{16} \mathrm{~cm}^{-3}$. In such structures, the QDs are filled with electrons. ${ }^{12,13}$ The independent capacitance-voltage measurements ${ }^{13}$ showed that on average two resident electrons occupy each QD at low temperature in the $n-i-n$ structure.

The carrier spin polarization was created by optical pumping with circular polarized light. ${ }^{14}$ A continuous wave tunable Ti:sapphire laser was used for excitation. The helicity of the exciting laser beam was alternated at $42 \mathrm{kHz}$ using a quartz photoelastic modulator. ${ }^{15,16}$ Changing the excitation 


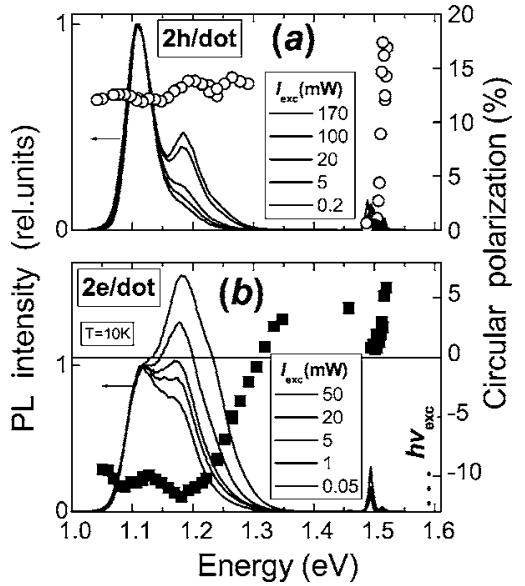

FIG. 1. PL (solid lines) and PL polarization spectra $(\bigcirc$ and $\mathbf{\square})$ of doubly positively (a) and negatively (b) charged delta-doped InAs QDs recorded at excitation into the GaAs barrier. $T=10 \mathrm{~K}$. Excitation energy $h \nu_{\text {exc }}(\mathrm{eV}): 1.653$ (a), 1.59 (b). Excitation power $I_{\text {exc }}(\mathrm{mW}): 1(\bigcirc), 5(\boldsymbol{\square})$.

helicity at a high frequency precluded dynamical polarization of spins of lattice nuclei. ${ }^{14,17}$ The spin polarization of charge carriers was determined by measuring the photoluminescence (PL) circular polarization degree, which is defined as $\rho=\left(I_{+}^{+}-I_{+}^{-}\right) /\left(I_{+}^{+}+I_{+}^{-}\right)$, where $I_{+}^{+}$and $I_{+}^{-}$are the intensities of the $\sigma^{+}$PL component under $\sigma^{+}$and $\sigma^{-}$pumping, respectively. To measure the PL circular polarization up to $1.4 \mu \mathrm{m}$, a highly sensitive polarization analyzer ${ }^{16}$ with an InGaAsP photomultiplier and a two-channel photon counter synchronized with the quartz polarization modulator was used. For the measurements in a longitudinal magnetic field, the sample was inserted in a center of a superconducting solenoid with a warm bore. The measurements were done at $10 \mathrm{~K}$.

PL and PL polarization spectra of the delta-doped QDs measured at excitation above the GaAs barrier are presented in Fig. 1. The solid lines in Fig. 1 show the PL spectra taken at different excitation intensities. The band centered at the $1.11 \mathrm{eV}$ is the PL of the QD ground state. We suppose that the high-energy shoulder within the range of $1.16-1.21 \mathrm{eV}$ is the radiation of the QD first excited state. With increasing excitation density, the shoulder grows due to the state-filling effect and even exceeds the ground-state PL in negatively charged dots. In positively charged QDs this effect is much less pronounced. This difference is most likely due to the following reasons. In InAs/GaAs QDs, the size quantization energy for electrons is much greater than for holes (50-70 meV against 5-10 $\mathrm{meV}$ between the ground and first excited states. ${ }^{18,19}$ ) Therefore, the visible "excited-state PL" is due to recombination of excited-state electrons. As in $n$-type QDs the ground state is filled with electrons, it takes lower pump power to have the excited electron level occupied for doubly negatively charged (2e) QDs than for doubly positively charged ( $2 h$ ) QDs. At high-excitation density, a weakly pronounced wing associated with the QD second excited state arises in the $1.22-1.26 \mathrm{eV}$ range indicating that there are at least three electron shells in the QD conduction band. The radiation near the $1.5 \mathrm{eV}$ comes from the GaAs barrier.

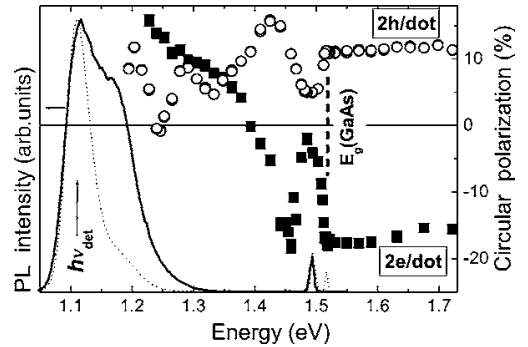

FIG. 2. PL and PL polarization excitation spectra of doublecharged delta-doped InAs QDs. $T=10 \mathrm{~K}$. PL spectra of the positively (dotted line) and negatively (solid line) charged dots recorded at $h \nu_{\text {exc }}=1.653 \mathrm{eV}, \quad I_{e x c}=0.2 \mathrm{~mW}, \quad$ and $h \nu_{\text {exc }}=1.59 \mathrm{eV}, \quad I_{\text {exc }}$ $=0.05 \mathrm{~mW}$, respectively. PL circular polarization excitation spectra of the positively $(\bigcirc)$ and negatively $(\boldsymbol{\square})$ charged dots measured in the center of the ground-state PL band. $h \nu_{\text {det }}(\mathrm{eV}): 1.109(\bigcirc), 1.117$ (ם). $I_{\text {exc }}(\mathrm{mW}): 5(\bigcirc), 20(\boldsymbol{\square})$.

The PL polarization spectra of positively and negatively charged dots are shown in Fig. 1 by open circles and closed squares, respectively.

PL circular polarization excitation spectra for positively $(\bigcirc)$ and negatively $(\boldsymbol{\square})$ charged dots measured in the center of the ground-state PL band are shown in Fig. 2. One can see in Figs. 1 and 2 that the polarization spectra are radically different for positively and negatively charged dots. Under above-barrier excitation, the ground-state PL polarization remains practically constant within a wide range of excitation energies $(1.52-1.73 \mathrm{eV})$ for both types of QDs, but it is negative for $2 e$-QDs and positive for $2 h$-QDs (see Fig. 2). This "sign rule" holds for excitation into the wetting layer also, but here the absolute value of polarization exhibits a strong nonmonotonous dependence on the excitation-photon energy, which is probably due to the splitting between heavy and light hole subbands in the wetting layer. Under excitation below the wetting layer $(<1.38 \mathrm{eV})$, the PL polarization in $2 e$-QDs becomes positive due to spin polarization of holes. ${ }^{20}$

In the following, we consider the case of above-barrier excitation. In this case the ground-state PL polarization is positive in positively charged dots and negative in negatively charged ones. To the contrary, the wetting-layer and barrier emissions are, of course, positively polarized for both types of QDs. This results in a crossover from negative to positive polarization in the PL spectra of negatively charged dots (at $\sim 1.32 \mathrm{eV}$ ), as seen in Fig. 1 .

The dependences of the ground-state PL polarization on excitation power and longitudinal magnetic field are also strongly different in negatively and positively charged dots. With increasing excitation intensity, the negative polarization rises from zero up to a saturation level, while the positive polarization always decreases, as shown in Fig. 3. We note that spectral dependences of $\rho$ and the dependence of $\rho$ on excitation power for doubly negatively charged QDs in a $n-i-n$ structure with one layer of InAs QDs (not shown here) are qualitatively similar to those shown in Figs. 1-3 for n-delta-doped dots.

Figure 4 shows that the negative polarization increases in weak magnetic fields applied in Faraday geometry, but strong fields suppress it. The positive polarization always increases as a function of magnetic field. 


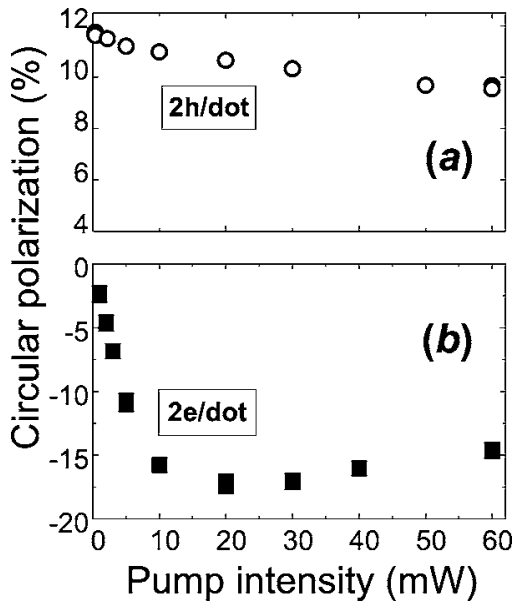

FIG. 3. Ground-state PL polarization vs excitation power for doubly positively (a) and negatively (b) charged InAs QDs. $T$ $=10 \mathrm{~K}$. (a) $h \nu_{e x c}=1.722 \mathrm{eV}, h \nu_{\mathrm{det}}=1.109 \mathrm{eV}$. (b) $h \nu_{e x c}=1.59 \mathrm{eV}$, $h \nu_{\mathrm{det}}=1.117 \mathrm{eV}$.

\section{THEORETICAL MODEL AND DISCUSSION}

\section{A. Qualitative explanation of the experimental results}

We start with a qualitative explanation of one of the main results of this work, the observation of opposite signs of the circular polarization of the ground-state PL in doubly positively charged $(2 h)$ and doubly negatively charged $(2 e)$ QDs under excitation in the barrier. In our opinion, the main reason for this difference is the fact that photoexcited electrons coming to QDs from the GaAs barrier retain their initial spin polarization $(50 \%)$, while holes completely depolarize before their capture into QDs. ${ }^{21}$ This is a consequence of a strong difference of spin relaxation times of holes and electrons in bulk semiconductors. ${ }^{14}$ As the ground-state PL of charged QDs is a result of recombination of a photoexcited carrier with a resident carrier of opposite charge and zero average spin polarization, one could expect positive polarization of

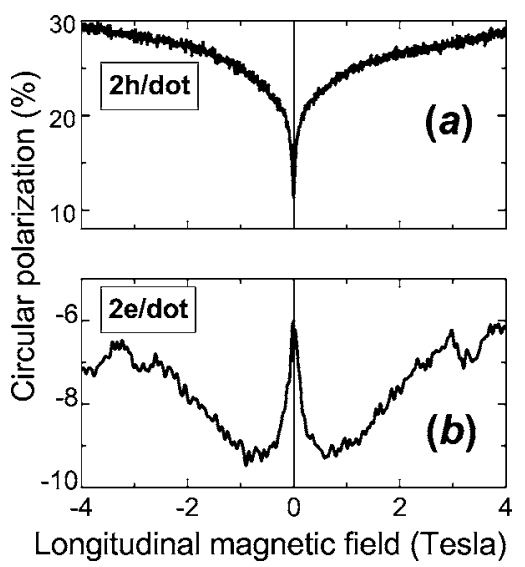

FIG. 4. Longitudinal magnetic field dependence of the groundstate PL polarization of doubly positively (a) and negatively (b) charged InAs QDs. $T=10 \mathrm{~K}$. Here, the sample with negatively charged dots is a $n-i-n$ structure with one layer of InAs QDs. (a) $h \nu_{e x c}=1.722 \mathrm{eV}, I_{e x c}=5 \mathrm{~mW}, h \nu_{\mathrm{det}}=1.109 \mathrm{eV}$. (b) $h \nu_{e x c}=1.55 \mathrm{eV}$, $I_{\text {exc }}=7 \mathrm{~mW}, h \nu_{\text {det }}=1.143 \mathrm{eV}$. the PL in positively charged QDs and no polarization of the PL in negatively charged QDs. In reality, however, the difference between positively and negatively charged QDs is even stronger, due to the anisotropic exchange (AE) electronhole interaction, which results in negative PL polarization in negatively charged QDs.

As shown earlier for excitons, ${ }^{3,7,22,23} \mathrm{AE}$ in an electronhole pair results in a simultaneous spin-flip transition of the electron and the hole. In other words, AE provides a transfer of angular momentum between the electron and the hole. Note, however, that "transfer" does not imply "conservation" in this case; a portion of the angular momentum goes to the crystal lattice via the spin-orbit interaction, making it possible to match the electron spin $1 / 2$ with the heavy hole spin $3 / 2$. In particular, this feature of the AE results in a possibility of flip-flop transitions for electron-hole pairs both with antiparallel and parallel spins. In the former case, similarly to the usual electron-electron spin exchange, the hole acquires a portion of angular momentum of the same sign as that lost by the electron. In the latter case, both the electron and the hole lose their angular momentum to the crystal lattice, so that the portion of angular momentum gained by the hole is opposite in sign to that lost by the electron. The matrix elements of these two transitions ( $\delta_{b}$ for the "bright" states with the total spin projection on the structure axis equal to \pm 1 and $\delta_{d}$ for the "dark" states with the spin projection \pm 2 ) are generally different. $^{23,24}$ If we now consider an ensemble of electronhole pairs where the electrons are spin-polarized and the holes are not, we can readily see that the electrons are in any case losing their average spin via AE, while the holes can acquire spin polarization of the same sign as that of electrons (if $\delta_{b}>\delta_{d}$ ), of the opposite sign (if $\delta_{b}<\delta_{d}$ ), or remain with zero spin polarization (if $\delta_{b}=\delta_{d}$ ). Theoretically, all these situations can be realized in $\mathrm{QDs}^{23,24}$; however, experimental works published up to now indicate that in real QD structures $\delta_{b} \gg \delta_{d}{ }^{7,22,24}$ Therefore, one can expect the AE to result in a spin polarization of holes parallel to the initial polarization of electrons. According to the well-known optical selection rules, ${ }^{14}$ the PL resulting from recombination of these holes with unpolarized resident electrons in negatively charged QDs has the helicity opposite to that of the exciting light. Such a "negative" PL polarization has been so far observed in singly negatively charged QDs. ${ }^{7,22}$ As the capture of an electron-hole pair into a singly charged QD results in the formation of a trion whose ground state is a Kramers spin doublet not affected by the AE, intermediate states were invoked to explain the negative polarization. According to Ref. 22 , the AE occurs in exciton before its binding with the resident electron. Cortez et al. ${ }^{7}$ explain their experimental results by the $\mathrm{AE}$ in the excited triplet state of the QD trion. In doubly charged QDs, two resident carriers form a spin singlet that does not hinder the $\mathrm{AE}$ in the photoexcited electron-hole pair. This fact, together with experimentally documented fast capture of photoexcited carriers from the barrier into QDs, suggests that the negative PL polarization observed in our experiments is due to the AE inside the QD. The basic properties of this interaction, including the relation between $\delta_{b}$ and $\delta_{d}$, should remain the same as for excitons, though values of these constants may be different. A theoretical model we propose on the basis of this assumption repro- 
duces all the main features of the observed phenomena, including the effects of magnetic fields and the dependence of the polarization on the excitation intensity both for positively and negatively doubly charged QDs. The model calculations are presented in Sec. III B. However, the main qualitative results of the model can be obtained without any mathematics.

Let us consider first the situation in 2e-QDs. If photoelectrons come from the barrier with spin down $(\downarrow)$, and photoholes come unpolarized (spin projection of the hole is $\Uparrow$ or $\Downarrow)$, then $|+1\rangle(\downarrow \uparrow \downarrow \uparrow)$ and $|-2\rangle(\downarrow \uparrow \downarrow \downarrow)$ tetrons are formed with equal probability. By analogy with the corresponding exciton states, we will in the following refer to $| \pm 1\rangle$ tetrons as bright, and to $| \pm 2\rangle$ tetrons as dark, though both these doublets are radiative. The emission of the $|+1\rangle$ bright tetron has $\sigma^{+}$polarization, while the $|-2\rangle$ dark tetron emits $\sigma^{-}$light. As the $\mathrm{AE}$ is stronger in the bright tetron $\left(\delta_{b} \gg \delta_{d}\right)$, its emission depolarizes faster, and therefore the net PL polarization is negative.

The magnetic field dependence of the polarization also finds its explanation in the different strength of the $\mathrm{AE}$ in bright and dark tetrons. Indeed, analogously to what has been observed earlier in excitons, ${ }^{22}$ the longitudinal magnetic field suppresses flip-flop transitions generated by the $\mathrm{AE}$ and restores polarization of both bright and dark tetrons. Besides, due to weaker $\mathrm{AE}$ in the dark tetron, its polarization is restored in weaker fields, and the magnitude of the negative PL polarization increases. Further increase of the magnetic field also suppresses the depolarization of the bright trion, and as a result the net PL polarization goes to zero in high magnetic field. This explains the experimentally observed $\mathrm{W}$-shaped magnetic field dependence of the PL polarization, shown in Fig. 4(b).

In $2 h$-QDs, the polarization of bright and dark tetrons has the same (positive) sign. Therefore, the increase of polarization of each of the two types of tetrons in the magnetic field should be accompanied by a monotonous increase of positive polarization of their net PL, as indeed is observed experimentally [see Fig. 4(a)].

In order to explain the experimentally observed dependence of the PL polarization on the pump intensity (see Fig. 3 ), the model should be expanded to include the capture kinetics of photoexcited electrons and holes. Based on the results of Refs. 21 and 25, we assume that under abovebarrier excitation electrons and holes are captured into QDs separately. Additionally, we suppose that a negatively charged QD first captures a hole and, only then, an electron (for positively charged dots the situation is opposite-an electron is captured first, then a hole). This assumption is based on a simple observation that a charged QD attracts particles of the opposite charge and repels like-charged ones and also on the experimental results of Ref. 26. An evident consequence is that the opposite-charge carrier lives for some time in the QD before the coming of the like-charge one; during this time interval, the QD contains not a tetron, but a trion. The stronger is pumping, the shorter is this time; at weak pumping, however, trions can exist for a long enough time to give a significant contribution into the photoluminescence. Therefore, the net PL of the QD ensemble is governed by trions at extremely low pumping and experi- ences a crossover to tetron-dominated regime at higher pump intensities (note that trion and tetron PL lines cannot be spectrally resolved in ensemble measurements). Now, as the ground-state trion PL has zero polarization for negatively charged QDs, and a polarization equal to that of photoexcited electrons in positively charged QDs, we can conclude that the net polarization as a function of pump intensity should be negative, increasing in the absolute value, in $2 e$-QDs, and positive, somewhat decreasing, in $2 h$-QDs. This behavior is indeed observed experimentally (see Fig. 3) and reproduced by model calculations in Sec. III B.

\section{B. Model calculations}

\section{Negatively charged QDs}

We assume that the photogenerated electrons and holes are captured into the dots separately under the above-barrier excitation. $^{21,25}$ Further, we believe that a photohole is captured first, forming a trion, and then a photoelectron is trapped to the QD first excited state, yielding a tetron. In accordance with the spin projection of electron $(\uparrow, \downarrow)$ and hole $(\Uparrow, \downarrow)$, we denote trion states as $\uparrow \downarrow \Uparrow$ and $\uparrow \downarrow \downarrow$, $|+1\rangle$ and $|-1\rangle$ tetrons as $\downarrow \uparrow \downarrow \Uparrow$ and $\uparrow \uparrow \downarrow \downarrow$, and $|+2\rangle$ and $|-2\rangle$ tetrons as $\uparrow \uparrow \downarrow \uparrow$ and $\downarrow \uparrow \downarrow \downarrow$. In the following, we will refer to $| \pm 1\rangle$ and $| \pm 2\rangle$ tetrons as bright and dark tetrons, respectively, in an analogy to bright and dark exciton states, though in fact both bright and dark tetron states are radiative and have equal oscillator strengths.

The photoexcited hole can recombine with the resident electron, either before or after the capture of a photoexcited electron.

The fraction of photons emitted by the QD with a photohole before capture of a photoelectron (trion emission) is

$$
\nu=\frac{1}{1+w \tau},
$$

where $\tau$ is the ground-state radiative lifetime; $w$ is an electron capture rate at the first excited level, which is proportional to the excitation intensity $I_{e x c}$. The polarization of this light is equal to the hole polarization $P_{h}$.

The fraction of photons emitted by the QD with a photohole after capture of a photoelectron via recombination of the photohole with one of the resident electrons (tetron emission) is

$$
\nu_{b}+\nu_{d}=\frac{w \tau}{1+w \tau},
$$

where $\nu_{b}$ and $\nu_{d}$ are the fractions of bright and the dark tetrons, formed by one $s$ hole, two $s$ electrons, and one $p$ electron.

As the holes are captured unpolarized, we put the fractions of bright and dark tetrons equal to each other

$$
\nu_{b}=\nu_{d}=\frac{1}{2} \frac{w \tau}{1+w \tau} .
$$

The net polarization of the QD emission is then given by the expression 


$$
\rho_{2 e}=\nu P_{h}+\nu_{b} P_{b}+\nu_{d} P_{d},
$$

where $P_{b}$ and $P_{d}$ are average polarizations of bright and dark tetrons. The initial polarization of the PL from bright and dark tetron states is determined by the polarization of captured photoelectrons $P_{e 0}: P_{b 0}=-P_{d 0}=P_{e 0}$, so that $\rho_{2 e}$ is initially equal to zero. It becomes nonzero due to unequal strength of the AE in the bright and dark tetron states, resulting in a disbalance of $P_{b}$ and $P_{d}$. In order to find these values at the moment of recombination, one should analyze the influence of the $\mathrm{AE}$ on the tetron spin state.

The AE interaction in the tetron, where the two electrons on the lowest level of size quantization are in a spin-singled state and therefore do not interact with other spins, is analogous to that in the exciton. The spin structure and dynamics

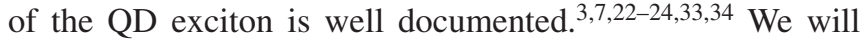
use the results of Refs. 22, 23, and 33 to analyze the polarization of tetron photoluminescence. This is done, briefly, as follows.

AE splits ground states of both the bright and the dark tetron into two levels with the wave functions being linear combinations of $+1 / 2$ and $-1 / 2$ states. This effect can be described by a two-level model with a pseudospin $1 / 2$, reflecting the tetron polarization, and an effective magnetic field of the AE directed perpendicularly to the initial direction of the pseudospin ( $z$ axis). An external magnetic field $B \| z$ splits pseudospin states with projections $+1 / 2$ and $-1 / 2$ onto the structure growth axis and for this reason, in this model, it is parallel to the initial pseudospin direction. A combined action of the two fields results in the pseudospin precession around their vector sum. Under a continuouswave excitation of tetrons, they are characterized by a pseudospin vector averaged over time (taking into account the lifetime of the corresponding tetron state). The PL circular polarization, determined by the $z$ projection of this vector, is given by the following expressions:

$$
\begin{gathered}
P_{b}=P_{e 0} \frac{1+\left(\Omega_{b z} \tau_{b}\right)^{2}}{1+\left(\Omega_{b z} \tau_{b}\right)^{2}+\left(\Omega_{b} \tau_{b}\right)^{2}}, \\
P_{d}=-P_{e 0} \frac{1+\left(\Omega_{d z} \tau_{d}\right)^{2}}{1+\left(\Omega_{d z} \tau_{d}\right)^{2}+\left(\Omega_{d} \tau_{d}\right)^{2}},
\end{gathered}
$$

similar to the formula for the electron mean spin in the Hanle effect in a tilted magnetic field. ${ }^{14}$ Here $\tau_{b, d}$ is the hole lifetime in bright and dark tetrons, $\hbar \Omega_{b z, d z}=g_{b, d} \mu_{B} B$ and $\hbar \Omega_{b, d}$ $=\delta_{b, d}$ are the Zeeman and anisotropic splittings of the bright and dark tetrons; $g_{b, d}$ is the $g$ factor of the bright or dark tetron and $\mu_{B}$ is the Bohr magneton.

Since the holes come from the barrier unpolarized $\left(P_{h}\right.$ $=0)$, then

$$
\begin{aligned}
\rho_{2 e}= & \frac{P_{e 0}}{2} \frac{w \tau}{1+w \tau}\left(\frac{1+\left(\Omega_{b z} \tau_{b}\right)^{2}}{1+\left(\Omega_{b z} \tau_{b}\right)^{2}+\left(\Omega_{b} \tau_{b}\right)^{2}}\right. \\
& \left.-\frac{1+\left(\Omega_{d z} \tau_{d}\right)^{2}}{1+\left(\Omega_{d z} \tau_{d}\right)^{2}+\left(\Omega_{d} \tau_{d}\right)^{2}}\right) .
\end{aligned}
$$

As, because of the symmetry of envelope wave functions, the ground-state hole recombines predominantly with one of the

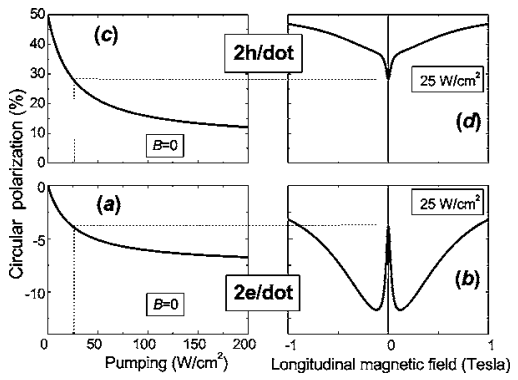

FIG. 5. Dependences of the PL circular polarization on the excitation intensity and longitudinal magnetic field in the double negatively and positively charged dots, calculated with Eqs. (6) and (8). The capture rate $w$ was calculated as $w=W / h v_{\text {exc }} N_{\text {dot }}$, where $W$ is the pump density and $N_{\text {dot }}$ is the dot density. The following parameters are used: $P_{e 0}=0.5, \quad \tau=0.5 \mathrm{~ns}, \quad\left|g_{b}\right|=3, \quad\left|g_{d}\right|=1.4, \quad \delta_{b}$ $=100 \mu \mathrm{eV}, \delta_{d}=2 \mu \mathrm{eV}, N_{\mathrm{dot}}=5 \times 10^{10} \mathrm{~cm}^{-2}, h v_{\text {exc }}=1.6 \mathrm{eV}$. (a) and (c) $B=0$. (b) and (d) $W=25 \mathrm{~W} / \mathrm{cm}^{2}$. See text for details.

ground-state electrons, we may take the recombination times of bright and dark tetrons, $\tau_{b}$ and $\tau_{d}$, approximately equal to each other, and replace them with a unique tetron lifetime $\tau$. In this case, in zero external field

$$
\rho_{2 e}=\frac{w \tau}{1+w \tau} \frac{P_{e 0}}{2}\left(\frac{1}{1+\left(\Omega_{b} \tau\right)^{2}}-\frac{1}{1+\left(\Omega_{d} \tau\right)^{2}}\right) .
$$

Since $\delta_{b} \gg \delta_{d},{ }^{7,22,24}$ the second term in Eq. (7) exceeds the first one, and $\rho_{2 e}$ is negative. The electron capture rate $w$ $\propto I_{\text {exc }}$. So, $\rho_{2 e} \propto I_{\text {exc }}$ at a weak pumping $w \tau \ll 1$ and goes to zero by decreasing the excitation intensity. With the excitation rise, the PL polarization achieves its maximal value that does not depend on the excitation intensity at $w \tau \gg 1$ and for a certain electron polarization $P_{e 0}$ is determined by the ratio of anisotropic splittings of bright and dark tetrons $\delta_{b}$ and $\delta_{d}$ [see Fig. 5(a)].

In these calculations we have assumed that the values of excitonic $g$ factors measured in Ref. $30\left(\left|g_{b}\right| \approx 3,\left|g_{d}\right| \approx 1.4\right)$ are suitable for our QDs also.

\section{Positively charged QDs}

We apply the same model to describe polarization in doubly positively charged dots. The difference is that now we assume that the photoelectron is captured first, forming a positively charged trion ( $\downarrow \Uparrow \downarrow$ or $\uparrow \Uparrow \downarrow)$. The subsequent capture of a photohole to the first excited state creates positively charged $(\downarrow \Uparrow \downarrow \downarrow \uparrow$ or $\uparrow \uparrow \downarrow \downarrow)$ and dark $(\downarrow \Uparrow \downarrow \downarrow$ or $\uparrow \uparrow \downarrow \uparrow)$ tetrons. Equations. (1)-(4) can be used to calculate the polarization, but now $\nu$ in Eq. (1) denotes the fraction of photons emitted by QDs with a photoelectron before the hole capture. Their polarization is $\rho=P_{e 0}$. Respectively, $w$ is a hole capture rate at the first excited level. By changing the sign in the expression $P_{d}$ in Eq. (5) (because the emission of dark tetrons in $2 h$-QDs is positively polarized), we obtain the following expression for the PL polarization in $2 h$ dots, $\rho_{2 h}=\nu P_{e 0}$ $+\nu_{b} P_{b}+\nu_{d} P_{d}$ : 


$$
\begin{aligned}
\rho_{2 h}= & P_{e 0} \frac{1}{1+w \tau}\left(1+\frac{w \tau}{2} \frac{1+\left(\Omega_{b z} \tau_{b}\right)^{2}}{1+\left(\Omega_{b z} \tau_{b}\right)^{2}+\left(\Omega_{b} \tau_{b}\right)^{2}}\right. \\
& \left.+\frac{w \tau}{2} \frac{1+\left(\Omega_{d z} \tau_{d}\right)^{2}}{1+\left(\Omega_{d z} \tau_{d}\right)^{2}+\left(\Omega_{d} \tau_{d}\right)^{2}}\right) .
\end{aligned}
$$

$\rho_{2 h}$ has the positive sign, because all the terms in Eq. (8) are positive. The maximal value of $\rho_{2 h}$ is equal to the initial polarization of electrons, $P_{e 0}$, which can be realized in the limit of weak pumping $\left(w \ll \tau^{-1}\right)$. With increasing intensity $\rho_{2 h}$ decreases and, at strong pumping $\left(w \gg \tau^{-1}\right)$ saturates at a level [Fig. 5(c)] that is determined, in the absence of the external magnetic field, by the ratio $\delta_{b} / \delta_{d}$. The longitudinal magnetic field always enhances the PL polarization, resulting in a V-shaped dependence $\rho_{2 h}(B)$ [see Fig. 5(d)]. The sharp increase of $\rho_{2 h}(B)$ at weak fields and much more smooth increase at higher fields are due to restoration of optical orientation in dark and bright tetrons, respectively.

\section{Discussion}

Dependences calculated for positively and negatively charged dots with the same set of parameters are shown in Fig. 5. One can see that the model calculation reflects all the main features of the experimental observations.

A W-shaped dependence of the negative PL polarization on the longitudinal magnetic field under above-barrier excitation has been observed earlier in singly charged $n$-doped InP quantum islands. ${ }^{22}$ Though the PL of the negative trion was detected, the dependence $\rho(B)$ was explained via magnetic-field-induced restoration of the polarization of photoexcited excitons before their binding with the resident electron and formation of a trion. For the weak AE in the dark exciton to result in a considerable loss of polarization, the exciton needs the lifetime in the island of at least $200 \mathrm{ps}^{22}$ This is possible in InP islands, having the lateral size 4-6 times larger than the exciton size $(\sim 100 \mathrm{~A})$. However, this scenario is not realistic in our InAs/GaAs QDs, because the capture of photoexcited carriers in such QDs is very fast ( $\leqslant 15 \mathrm{ps}$ ), ${ }^{7,21}$ the dot size is comparable to the Bohr radius of the exciton, and, as a consequence, photoexcited and resident carriers immediately form a complex.

Further corrections to the model should involve multiexcitonic effects, which can be considerable at high excitation power. However, we believe that the essential features of the observed phenomena are captured by the simplest tetron model. Some other possible effects are discussed below.

\section{Electron spin relaxation due to hyperfine interaction}

In doubly negatively charged QDs, the photoexcited $(p)$ electron is coupled with the lattice nuclei by hyperfine interaction (on the contrary, hyperfine interaction of holes with lattice nuclei is absent ${ }^{27}$ ), and its polarization can be destroyed as a result of a precession of its spin in a fluctuation of the effective nuclear field. ${ }^{5}$ However, the isotropic part of its exchange interaction with the hole, which splits the spin states of the tetron into bright and dark doublets, suppresses the precession of the electron spin. An estimation with Eq. (7) of Ref. 5 gives the energy of the hyperfine interaction of an electron with the nuclear spin fluctuation $\delta_{\mathrm{eN}} \sim 5 \mu \mathrm{eV}$ (we assume that in our samples an InAs QD contains $\approx 10^{4}$ nuclei, the spins and the hyperfine constants of indium and arsenic are equal to $I_{\mathrm{In}}=9 / 2, I_{\mathrm{As}}=3 / 2, A_{\mathrm{In}}$ $\approx 56 \mu \mathrm{eV}, A_{\mathrm{As}} \approx 46 \mu \mathrm{eV}$, respectively). ${ }^{28,29}$ We suppose that the exchange interaction between the $p$ electron and the $s$ hole in the tetron is of the same order as the exchange interaction in the ground $(s-s)$ exciton state. The exchange splitting of bright and dark exciton states, $\delta_{0}$, is $100-600 \mu \mathrm{eV}$ in InAs/GaAs QDs, ${ }^{4,24,30,31}$ which is considerably higher than the hyperfine energy $\delta_{\mathrm{eN}}$. Therefore, the electron spin-flip transitions due to the hyperfine interaction are suppressed in the negative tetron (a stabilization of the electron spin by the exchange interaction was earlier considered for the exciton in Ref. 32).

In a doubly positively charged $\mathrm{QD}$, the photoelectron comes first and, before the hole capture, experiences the influence of the nuclear fluctuation. In this case, the degree of depolarization of electrons is determined by the relations of the dephasing time of the electron ensemble in fluctuation nuclear fields, $T_{\Delta}$, with the electron recombination time with resident holes, $\tau$, and the capture time of the hole, $w^{-1}$. The latter can vary within a wide range depending on the pump intensity. In the InAs QDs under study, $T_{\Delta} \approx 0.2 \mathrm{~ns},{ }^{35} \tau$ $\sim 1 \mathrm{~ns}^{4,7,21}$ At weak pumping, when the hole capture rate is small, $T_{\Delta}$ is the shortest time, and the nuclear-induced electron spin dephasing may dominate. In this case, for zero external field, one can use Eq. (8) with the initial electron polarization $P_{e 0}$ replaced by $P_{e 0} / 3$. This does not qualitatively change the calculated dependence of $\rho_{2 h}$ on pump intensity, plotted in Fig. 5(c).

Magnetic fields should suppress the effect of nuclear fluctuations and, consequently, increase the electron polarization. Since the energies of the hyperfine interaction $\delta_{\mathrm{eN}}$ and the AE in the dark tetron $\delta_{d}$ are comparable, suppression of both these interactions can give rise to a sharp increase of the PL polarization observed in weak magnetic field [Fig. 4(a)]. We would like to note that the suppression of nuclear fluctuations by a magnetic field should be more pronounced at weak pumping, where the depolarizing effect of nuclear fluctuations is stronger.

\section{Spin memory effects}

Our model does not consider the effects of "spin memory," i.e., of the spin polarization of resident electrons remaining after recombination of a polarized electron-hole pair in $2 e$-QDs. After recombination of the dark tetron, the QD remains with two electrons having the same spin direction (predominantly $\downarrow \downarrow$ in the case of $\sigma^{+}$excitation). This is again a doubly charged QD and, as we suggested above, it should capture a hole first. Since photoexcited holes are not polarized, holes $\Uparrow$ and $\Downarrow$ can be captured with equal probabilities. The $\Uparrow$ hole forms a hot trion $\downarrow \downarrow \Uparrow$, emitting $\sigma^{+}$light. However, AE of the $\Uparrow$ hole and each of the two $\downarrow$ electrons is strong and can transform the hot trion into the cold one with the spin configuration $\downarrow \uparrow \downarrow,{ }^{7}$ which lives long and emits $\sigma^{-}$ light increasing the negative PL polarization. A capture of the $\Downarrow$ hole forms a hot dark trion $\downarrow \downarrow \Downarrow$. The AE in such a trion would result in a cold trion $\downarrow \uparrow \uparrow$, producing the $\sigma^{+}$emission. 
But the $\mathrm{AE}$ in dark trions is weaker than in bright ones; therefore, the net PL polarization of $\downarrow \downarrow$ QDs after a hole capture is, presumably, $\sigma^{-}$.

Before the hole capture, the two $\downarrow \downarrow$ electrons may be affected by the hyperfine interaction with a nuclear spin fluctuation, which would deflect their total spin from its initial direction. As a result, within the time comparable to the precession period of an electron spin in the nuclear-fluctuation field $\left(T_{\Delta} \approx 0.2 \mathrm{~ns}\right),{ }^{35}$ these QDs, retaining the total spin of the two electrons equal to 1 , will have zero projection of the total spin onto the structure growth axis (a more detailed consideration of this process will be published elsewhere). After the hole capture, such dots will give unpolarized emission.

Thus, the QDs with two spin-polarized electrons yield on average $\sigma^{-}$light. As the relative number of such dots is small, their contribution should not considerably affect the PL polarization of the whole ensemble of QDs.

To summarize, we observed optical spin orientation in doubly negatively and positively charged quantum dots. We propose a theoretical model including separate capture of photoexcited electrons and holes from the barrier and the anisotropic exchange interaction in doubly charged excitons (tetrons). The model explains different signs of the circular polarization of the emission from the ground state of positively and negatively charged dots, as well as the polarization dependence on the pump intensity and the magnetic field in the Faraday geometry.

\section{ACKNOWLEDGMENTS}

This work was partially supported by Nanoscience Special Project of University of Tsukuba, INTAS (Grant No. 1B 2167), RFBR, the Russian Academy of Sciences program, and Program of the Russian Ministry of Science and Technology. One of the authors (I.A.M.) thanks CRDF. The authors are grateful to R.I. Dhzioev, V.L. Korenev, M.M. Afanasiev, X. Marie, and T. Amand for useful discussions.
*Corresponding author. Fax: (+7)-(812)-2471017. Email address: kalevich@solid.ioffe.ru

${ }^{1}$ A. Abragam and B. Bleaney, Electron Paramagnetic Resonance of Transition Ions (Clarendon Press, Oxford, 1970).

${ }^{2}$ A. V. Khaetskii and Y. V. Nazarov, Phys. Rev. B 61, 12639 (2000).

${ }^{3}$ R. I. Dzhioev, B. P. Zakharchenya, E. L. Ivchenko, V. L. Korenev, Yu. G. Kusraev, N. N. Ledentsov, V. M. Ustinov, A. E. Zhukov, and A. F. Tsatsul'nikov, JETP Lett. 65, 804 (1997).

${ }^{4}$ M. Paillard, X. Marie, P. Renucci, T. Amand, A. Jbeli, and J. M. Gérard, Phys. Rev. Lett. 86, 1634 (2001).

${ }^{5}$ I. A. Merkulov, Al. L. Efros, and M. Rosen, Phys. Rev. B 65, 205309 (2002).

${ }^{6}$ I. E. Kozin, V. G. Davydov, I. V. Ignatiev, A. V. Kavokin, K. V. Kavokin, G. Malpuech, Hong-Wen Ren, M. Sugisaki, S. Sugou, and Y. Masumoto, Phys. Rev. B 65, 241312(R) (2002).

${ }^{7}$ S. Cortez, O. Krebs, S. Laurent, M. Sénès, X. Marie, P. Voisin, R. Ferreira, G. Bastard, J.-M. Gérard, and T. Amand, Phys. Rev. Lett. 89, 207401 (2002); T. Amand, B. Urbaszek, M. Sénès, P. Renucci, X. Marie, O. Krebs, S. Laurent, S. Cortez, P. Voisin, R. J. Warburton, B. D. Gerardot, P. M. Petroff, and J.-M. Gérard, Proceedings of PSSN2003, Kyoto, Japan (unpublished); http:// www.ipc.kit.ac.jp/ takaghra/pssn.html

${ }^{8}$ I. V. Ignatiev, I. Ya. Gerlovin, M. Ikezawa, V. K. Kalevich, S. Yu. Verbin, and Y. Masumoto, Physica E (Amsterdam) 17, 361 (2002).

${ }^{9}$ T. Flissikowski, I. A. Akimov, A. Hundt, and F. Henneberger, Phys. Rev. B 68, 161309(R) (2003).

${ }^{10}$ D. Bimberg, M. Grundmann, and N. N. Ledentsov, Quantum Dot Heterostructures (Wiley, Chichester, 1999).

${ }^{11}$ V. M. Ustinov, A. E. Zhukov, A. Yu. Egorov, and N. A. Maleev, Quantum Dot Lasers (Oxford University Press, Oxford, 2003).

${ }^{12}$ V. K. Kalevich, M. Ikezawa, T. Okuno, A. Yu. Shiryaev, A. E. Zhukov, V. M. Ustinov, P. N. Brunkov, and Y. Masumoto, Phys. Status Solidi B 238, 250 (2003).
${ }^{13}$ P. N. Brunkov, A. A. Suvorova, N. A. Bert, A. R. Kovsh, A. E. Zhukov, A. Yu. Egorov, V. M. Ustinov, A. F. Tsatsul'nikov, N. N. Ledentsov, P. S. Kop'ev, S. G. Konnikov, L. Eaves, and P. S. Main, Semiconductors 32, 1096 (1998).

${ }^{14}$ Optical Orientation, edited by F. Meier and B. Zakharchenya, Modern Problems in Condensed Matter Sciences Vol. 8 (NorthHolland, Amsterdam, 1984).

${ }^{15}$ S. N. Jasperson and S. E. Schnatterly, Rev. Sci. Instrum. 40, 761 (1969).

${ }^{16}$ V. D. Kul'kov and V. K. Kalevich, Instrum. Exp. Tech. 24, 1265 (1981).

${ }^{17}$ Alternating the helicity of the exciting light results in periodic change of the mean spin direction of photoexcited electrons. As the modulation period is much shorter than the longitudinal spin relaxation time of the nuclear spin, the nuclei cannot follow the rapidly changing direction of the electron spin, and no dynamical nuclear spin polarization develops.

${ }^{18}$ O. Stier, M. Grundmann, and D. Bimberg, Phys. Rev. B 59, 5688 (1999).

${ }^{19}$ A. J. Williamson, L. W. Wang, and Alex Zunger, Phys. Rev. B 62, 12963 (2000).

${ }^{20}$ V. K. Kalevich, M. Ikezawa, T. Okuno, A. Yu. Shiryaev, K. V. Kavokin, P. N. Brunkov, A. E. Zhukov, V. M. Ustinov, and Y. Masumoto, Physica E (Amsterdam) 21, 1018 (2004).

${ }^{21}$ V. K. Kalevich, M. Paillard, K. V. Kavokin, X. Marie, A. R. Kovsh, T. Amand, A. E. Zhukov, Yu. G. Musikhin, V. M. Ustinov, E. Vanelle, and B. P. Zakharchenya, Phys. Rev. B 64, 045309 (2001).

${ }^{22}$ R. I. Dzhioev, B. P. Zakharchenya, V. L. Korenev, P. E. Pak, D. A. Vinokurov, O. V. Kovalenkov, and I. S. Tarasov, Phys. Solid State 40, 1587 (1998).

${ }^{23}$ G. E. Pikus and E. L. Ivchenko, Superlattices and Other Heterostructures, Springer Series in Solid-State Sciences Vol. 110 (Springer-Verlag, Berlin, 1995).

${ }^{24}$ M. Bayer, G. Ortner, O. Stern, A. Kuther, A. A. Gorbunov, A. 
Forchel, P. Hawrylak, S. Fafard, K. Hinzer, T. L. Reinecke, S. N. Walck, J. P. Reithmaier, F. Klopf, and F. Schäfer, Phys. Rev. B 65, 195315 (2002).

${ }^{25}$ M. Grundmann and D. Bimberg, Phys. Rev. B 55, 9740 (1997).

${ }^{26}$ K. Gündogdu, K. C. Hall, Tomas F. Boggess, D. G. Deppe, and O. B. Shchekin, Appl. Phys. Lett. 84, 2793 (2004).

${ }^{27}$ E. I. Gr'ncharova and V. I. Perel', Sov. Phys. Semicond. 11, 997 (1977).

${ }^{28}$ M. Gueron, Phys. Rev. 135, A200 (1964).

${ }^{29}$ D. Paget, G. Lampel, B. Sapoval, and V. I. Safarov, Phys. Rev. B 15, 5780 (1977).

${ }^{30}$ M. Bayer, A. Kuther, A. Forchel, A. Gorbunov, V. B. Timofeev, F. Schäfer, J. P. Reithmaier, T. L. Reinecke, and S. N. Walck, Phys. Rev. Lett. 82, 1748 (1999).
${ }^{31}$ B. Urbaszek, R. J. Warburton, K. Karrai, B. D. Gerardot, P. M. Petroff, and J. M. Garcia, Phys. Rev. Lett. 90, 247403 (2003).

${ }^{32}$ D. Gammon, Al. L. Efros, T. A. Kennedy, M. Rosen, D. S. Katzer, D. Park, S. W. Brown, V. L. Korenev, and I. A. Merkulov, Phys. Rev. Lett. 86, 5176 (2001).

${ }^{33}$ R. I. Dzhioev, H. M. Gibbs, E. L. Ivchenko, G. Khitrova, V. L. Korenev, M. N. Tkachuk, and B. P. Zakharchenya, Phys. Rev. B 56, 13405 (1997).

${ }^{34}$ D. Gammon, Al. L. Efros, J. G. Tischler, A. S. Bracker, V. L. Korenev, and I. A. Merkulov, Chapter 6 in Quantum Coherence, Correlation and Decoherence in Semiconductor Nanostructures, edited by T. Takagahara (Academic Press, Amsterdam, 2002).

${ }^{35}$ An estimation with Eq. (11) of Ref. 5 gives $T_{\Delta} \approx 0.2 \mathrm{~ns}$ for our InAs QDs. 